\title{
Universality of Entanglement Transitions from Stroboscopic to Continuous Measurements
}

\author{
M. Szyniszewski® ${ }^{1,2, *}$ A. Romito, ${ }^{1}$ and H. Schomerus ${ }^{1}$ \\ ${ }^{1}$ Department of Physics, Lancaster University, Lancaster LA1 4YB, United Kingdom \\ ${ }^{2}$ Department of Physics and Astronomy, University College London, London WCIE 6BT, United Kingdom
}

(Received 12 May 2020; revised 14 August 2020; accepted 22 October 2020; published 20 November 2020)

\begin{abstract}
Measurement-driven transitions between extensive and subextensive scaling of the entanglement entropy receive interest as they illuminate the intricate physics of thermalization and control in open interacting quantum systems. While this transition is well established for stroboscopic measurements in random quantum circuits, a crucial link to physical settings is its extension to continuous observations, where for an integrable model it has been shown that the transition changes its nature and becomes immediate. Here, we demonstrate that the entanglement transition at finite coupling persists if the continuously measured system is randomly nonintegrable, and show that it is smoothly connected to the transition in the stroboscopic models. This provides a bridge between a wide range of experimental settings and the wealth of knowledge accumulated for the latter systems.
\end{abstract}

DOI: 10.1103/PhysRevLett.125.210602

Subjecting a complex quantum system to observations can have drastic effects on its time evolution. The most celebrated example is the quantum Zeno effect [1-3], according to which continuous projective measurements can freeze the dynamics of a quantum system completely. Recent work has established [4-8] and developed [9-39] an illuminating extension of this effect, where the quantum dynamics change in a phase transition when stroboscopic measurements occur with sufficient strength or frequency. This transition is manifested in the entanglement characteristics of the system, as captured by the entanglement entropy

$$
S=\operatorname{tr}\left(\rho_{A} \ln \rho_{A}\right)
$$

with the reduced density matrix of a subsystem $A$. In the transition, the entropy changes its scaling with the system size [5,40-45] from extensive, indicating ergodic manybody dynamics, to subextensive, signaling localization of the underlying quantum-coherent correlations.

A key question to make this rapidly growing body of knowledge on stroboscopic systems applicable to physical settings is the fate of the entanglement transition for continuous variable-strength observations. These not only more accurately reflect the reality of many experimental architectures [46-55], but also enable us to apply this knowledge to the generic effects of coupling to an environment that may not per se have been designed to carry out a measurement. For an integrable system, it has been shown that the transition can indeed completely change its nature when observations become continuous, in that it then can occur at infinitesimal small measurement strengths [56].
Here, we show that the transition from the stroboscopic models is reinstated for continuous observations of a randomly evolving, nonintegrable, system. We achieve this by formulating a model that allows us to interpolate between a stroboscopic random circuit and a continuously evolving one, and trace the entanglement characteristics numerically in terms of the entanglement entropy and mutual information. The established link between these limits lends further relevance to deep results arrived for the stroboscopic circuits-such as emerging conformal symmetry $[6,7,9-11]$ as well as approximations that permit us to reach very large system sizes $[4,5,7,31-34,57,58]$ giving them direct bearing on a much wider range of experimental settings.

Model.-We base our modeling on the universal quantum-circuit architecture [5,59-61] shown in Fig. 1, which

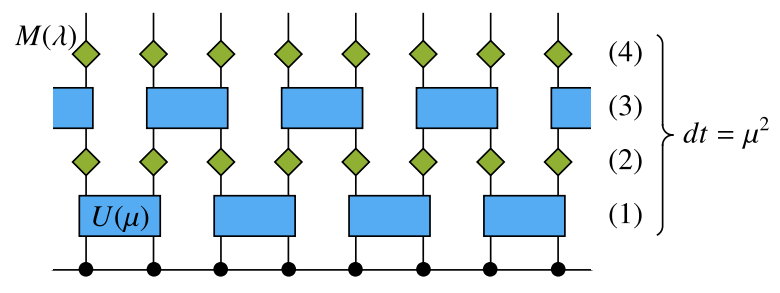

FIG. 1. We study the entanglement dynamics in a random circuit model, combining unitary evolutions $U$ and measurements $M$ such that one can interpolate between the continuum limit ( $U$ near the identity matrix and measurements weak) and widely studied fully random, stroboscopic models. This is achieved by equipping the unitary matrices with a parameter $\mu$ that determines the physical timescale of the dynamics according to $d t \sim \mu^{2}$, and the measurements with a parameter $\lambda$ so that the effective measurement strength is given by $\lambda_{0}=\lambda / \mu$. 
describes the dynamics of $L$ spins (dots) evolving under the action of unitary gates $U$ (rectangles) and nonunitary measurement operations $M$ (diamonds) on individual spins. Two layers of gates and measurements make up one time step $d t$ and iteration over $n$ steps induces a discrete time evolution of the quantum state $\left|\psi_{n}\right\rangle$. In the original design [4-6], the gates are completely random, according to unitary matrices $U$ drawn from a circular ensemble with probability distribution given by the corresponding Haar measure, while the measurements are projective, so that the time step $d t=\mathrm{O}(1)$ in terms of physical timescales governing the dynamics. This design can be easily adapted to other situations, including systems with deterministic dynamics $[24-28,36,56]$ or other types of measurements $[8,11,22,23,28,36]$.

Here, we carry out two such modifications designed to change the dynamics and observation strength over the timescale $d t$, thereby allowing us to take the continuum limit in which $d t \rightarrow 0$.

(a) The unitary matrices $U$ are generated from an ensemble parametrized by $0 \leq \mu \leq 1$, which interpolates between matrices close to the identity matrix $(\mu \ll 1)$ and the exact Haar measure $(\mu=1)$. This is realized using the Poisson kernel [62],

$$
U=\left(V+\sqrt{1-\mu^{2}} \mathbb{1}\right)\left(\mathbb{1}+\sqrt{1-\mu^{2}} V\right)^{-1},
$$

where $V$ is a random unitary matrix distributed according to the Haar measure. The latter is recovered for $\mu=1$, where $U=V$. For $\mu \ll 1$, the matrices localize close to the identity matrix,

$$
U \approx \mathbb{1}-i d t H_{\mathrm{eff}}, \quad d t \equiv \mu^{2}, \quad H_{\mathrm{eff}}=\frac{i}{2}(V-\mathbb{1})(V+\mathbb{1})^{-1},
$$

which identifies the Cayley transform of $V$ as the effective Hamiltonian $H_{\text {eff }}$, and sets the physical timescale according to the resulting stable Cauchy process [63]. However, this Wiener process does not permit the exact extrapolation to the stroboscopic case. Between these limits, the matrices preserve unitarity and maintain randomness according to a probabilistic maximal-entropy principle [64,67].

(b) The projective measurements are replaced by weak measurements, implemented by coupling the $z$ component of a given spin to an external pointer with a continuous readout $x$, prepared initially in a Gaussian state. Measurements are of strength $\lambda$, ranging from the case of no measurements $(\lambda=0)$ to the standard case of projective measurements $(\lambda \rightarrow \infty)$. These take the form of positive-operator-value measurements [68,69] with Kraus operators

$$
M(x)=G(x-\lambda) \Pi_{i}^{+}+G(x+\lambda) \Pi_{i}^{-},
$$

where $G(x)=\exp \left(-x^{2} / 2\right) / \pi^{1 / 4}$ is a Gaussian of unit width centered around zero, and $\Pi_{i}^{ \pm}=\left(1 \pm \sigma^{z}\right)_{i} / 2$ are projection operators onto spin-up or spin-down on site $i$. For a given readout $x$, the system state is updated via

$$
|\psi\rangle \rightarrow \frac{1}{\sqrt{P(x)}} M(x)|\psi\rangle,
$$

where $P(x)=\left\langle\psi\left|M(x)^{\dagger} M(x)\right| \psi\right\rangle$ is the probability distribution of the measurement output. For small $\lambda$, the measurement model reduces to a generic Wiener process

$$
|\psi\rangle \rightarrow \mathcal{N}\left[1-\sum_{i}\left(\lambda^{2}\left\langle\sigma_{i}^{z}\right\rangle+\delta W_{i}\right) \sigma_{i}^{z}\right]|\psi\rangle,
$$

where the random variables $W_{i}$ are independently Gaussian distributed with zero mean and variance $\lambda^{2}$, and $\mathcal{N}$ is a normalization constant.

Writing the intrinsic scale of this process as $\lambda^{2}=\lambda_{0}^{2} d t=\lambda_{0}^{2} \mu^{2}$, the effective strength of the measurement in our model is therefore given by

$$
\lambda_{0}=\lambda / \mu,
$$

which has to be kept fixed as we send $d t=\mu^{2} \rightarrow 0$. The physical timescale for the dynamics is then given by $t=n d t=n \mu^{2}$, where $n$ is the number of steps through the circuit depicted in Fig. 1. Our main result will be to establish that an entanglement transition occurs at a finite value of $\lambda_{0}$, for all scenarios from the continuum limit to the fully random stroboscopic case.

Entanglement dynamics in the continuum limit.-Figure 2 illustrates the effect of the described modifications on the entanglement dynamics in terms of the entanglement entropy for a subsystem of size $L / 2$, averaged over 1000 realizations of the dynamics initialized to a Néel state. Time is measured as $t=n \mu^{2}$; in each panel, $\lambda_{0}=\lambda / \mu$ is kept fixed, while each curve corresponds to a different value of $\mu$.

In all cases, the entanglement entropy initially increases with time, but then saturates in a quasistationary regime, at a measurement-strength dependent value $S_{\infty}$ that we will analyze further in the pursuit of the entanglement transition. For the moment, the key point in the figure is the collapse of curves at $\mu \lesssim 0.1$, which therefore delineates the continuum regime. That this collapse occurs both for the rate of entanglement spreading as well as for the saturation value confirms the described scaling of time and measurement strength in this regime. Outside of the continuum regime, the entanglement dynamics display a notable dependence on $\mu$, both in the rate of initial entanglement spreading as well as for the saturation value, aspects to which we return later when we discuss the connection to the stroboscopic case. First, we establish that an entanglement transition occurs in the continuum regime, for which we set $\mu=0.05$ (equivalently, $d t=0.0025$ ).

This is demonstrated in Fig. 3, which shows the average and variance of the saturation value $S_{\infty}$ of the entanglement 


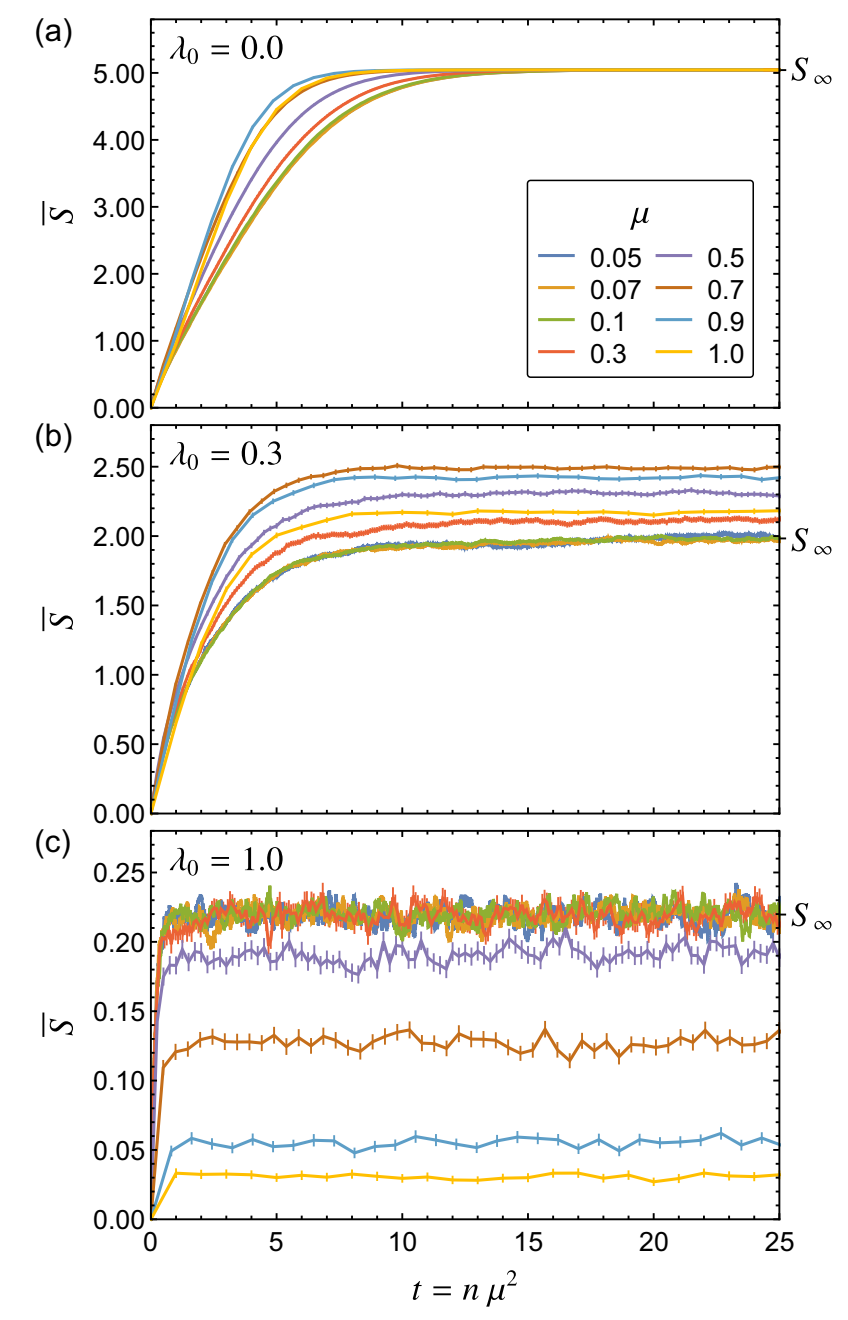

FIG. 2. Entanglement dynamics in the random-circuit model of Fig. 1, as captured by the time evolution of the averaged bipartite entanglement entropy $\bar{S}$. Time is measured in units $t=n \mu^{2}$, the system size is $L=16$, and results are averaged over 1000 realizations. The different panels fix the effective measurement strength to (a) $\lambda_{0}=0$, (b) $\lambda_{0}=0.3$, and (c) $\lambda_{0}=1.0$, with the different curves corresponding to different choices of $\mu$. Throughout the whole dynamics, the curves collapse for $\mu \lesssim 0.1$, which indicates entering the continuum regime. Increasing the measurement strength suppresses the quasistationary value $S_{\infty}$, which raises the question of an entanglement transition addressed in the subsequent figures.

entropy for different system sizes $L$ as a function of the measurement strength $\lambda_{0}$. As seen in panel (a), the entanglement entropy is large and increases with system size when the measurement strength is small, but drops to a small, system-size-independent value when the measurement strength is large. As further illustrated in the inset, this qualitative change of the scaling occurs in the range $0.2<\lambda_{0}<0.4$. Panel (b) shows that the sample-to-sample fluctuations $\operatorname{var} S_{\infty}$ indeed become large in this range. While the position $\lambda_{0}^{\max }$ where the fluctuations are maximal drifts to smaller values as $L$ is increased, its extrapolation to infinite system size (inset) yields a finite critical value

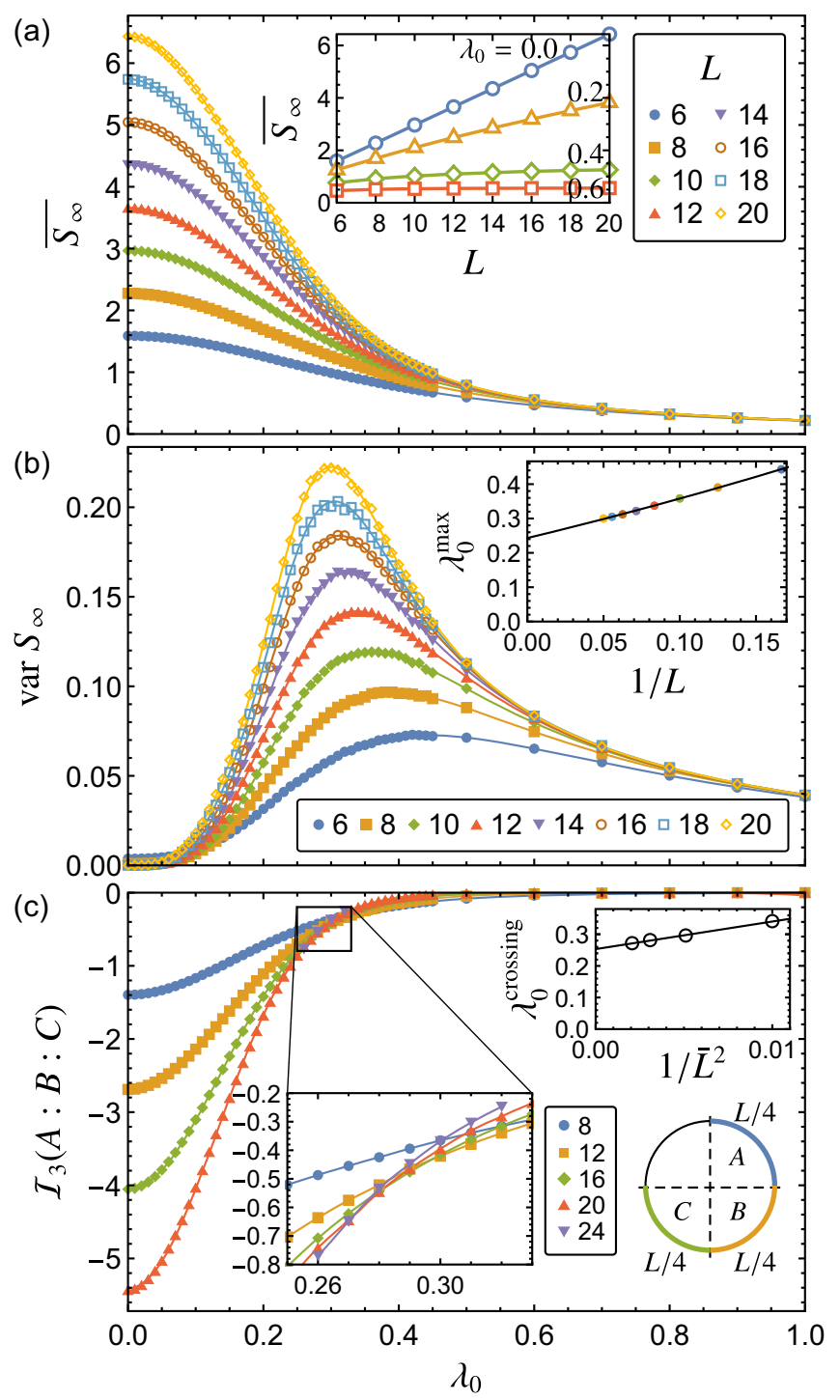

FIG. 3. (a) Average saturation entropy $\overline{S_{\infty}}$ and (b) corresponding fluctuations var $S_{\infty}$, as a function of measurement strength $\lambda_{0}$ for different system sizes $L$. The inset in (a) shows $\overline{S_{\infty}}$ for fixed $\lambda_{0}$ as $L$ is increased, while the inset (b) shows the extrapolation of the position $\lambda_{0}^{\max }$ of maximal variance to an infinite system size. (c) Tripartite mutual information $\mathcal{I}_{3}(A: B: C)$ as a function of measurement strength $\lambda_{0}$ for different system sizes $L$, where the subsystems are all of size $L / 4$, as indicated in the bottom right inset. The left inset focuses on the region where the curves cross, while the top right inset shows the extrapolation of the crossing positions to an infinite system size, where $\bar{L}$ is the average of the system sizes for which the crossing occurs.

$\lambda_{0}^{\text {crit }} \approx 0.243(4)$. Using this critical value for finite-size scaling yields the critical exponent of the correlation length $\nu=0.70(1)$ [65]. Panel (c) provides further evidence for the transition in terms of the tripartite mutual information

$$
\begin{aligned}
\mathcal{I}_{3}(A: B: C)= & S(A)+S(B)+S(C)+S(A \cup B \cup C) \\
& -S(A \cup B)-S(A \cup C)-S(B \cup C),
\end{aligned}
$$


defined for three such subsystems $A, B$, and $C$. Here, the transition is indicated by the crossing point, which has been found to show reduced finite-size effects in the original stroboscopic model [17]. As shown in the insets, these features also hold in the present model, with the position of the crossings approaching a critical value of $\lambda_{0}^{\text {crit }}=0.253(2)$ that agrees well with the transition point obtained using the variance analysis [65].

Connection to the stroboscopic case.-Having established the entanglement transition in the continuum regime, we now come to the second main point of this Letter, namely, its connection to the transition in the original stroboscopic model. This is afforded in our model by being able to tune the timescale $d t=\mu^{2}$ from 0 to 1 . Returning to Fig. 2, outside the continuum regime the measurements still have the effect to suppress the saturation entropy, but down to even smaller, $\mu$-dependent, values. For a detailed analysis, Fig. 4(a) shows how the saturation entanglement entropy changes with $\mu$ for fixed $\lambda_{0}$, where differently articulated curves correspond to different system sizes $L$. Depending on the measurement strength, we find two scenarios. For $\lambda_{0} \lesssim 0.4$, the saturation entropy remains
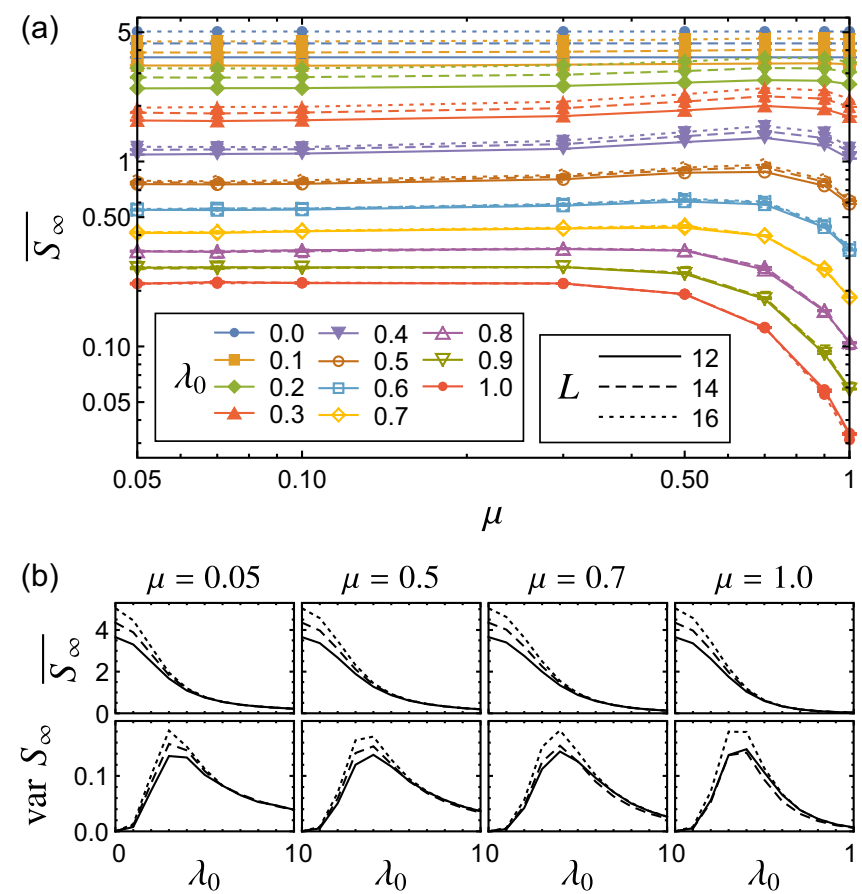

FIG. 4. (a) Averaged saturation value $\overline{S_{\infty}}$ of the entanglement entropy for different values of $\mu$ in the whole range from the continuum limit $(\mu \rightarrow 0)$ to the fully random stroboscopic case $(\mu=1)$. In each curve, the measurement strength $\lambda_{0}=\lambda / \mu$ and the system size are kept fixed. Across the whole range of $\mu$, the entanglement entropy changes its scaling from extensive to subextensive around $\lambda_{0} \approx 0.3$. As shown in the subpanels in (b), the qualitative entanglement characteristics in the continuum regime [Figs. 3(a) and 3(b)] indeed also occur for intermediate values $(\mu=0.5$ and $\mu=0.7$ ), with $\mu=1$ reproducing the conventional stroboscopic case. essentially $\mu$ independent, and shows a systematic system-size dependence with an extensive scaling, corresponding to ergodic behavior. For larger measurement strengths, on the other hand, the entropy displays the above-mentioned downturn as one approaches the stroboscopic limit—but also becomes independent of the system size across the whole parameter range. As shown in the subpanels in (b), in the intermediate range between the continuum regime and the stroboscopic case, the average and fluctuations of the entanglement entropy display the same qualitative behavior as in Fig. 3, with an only weak $\mu$ dependence of the critical value $\lambda_{0}^{\text {crit. }}$. These results demonstrate a substantial degree of universality of the entanglement transition in the whole range from the continuum regime to the fully random stroboscopic case.

Conclusions.-In summary, we showed that measurement-driven entanglement transitions can occur in continuously evolving and monitored systems. We established this in a flexible extension of random-circuit models, by which we could directly relate the transition to the widely studied stroboscopic case. This uncovered a significant degree of university in the entanglement dynamics. As we show in Ref. [65], this universality further extends to the variation of the measurement frequency $p$ (the parameter that was varied in the original studies of the stroboscopic model), where results remain invariant upon a simple rescaling $\lambda_{0}=\sqrt{p} \lambda / \mu$ of the effective measurement strength. In this way, results derived for stroboscopic models gain a much larger range of applicability.

The model described in this Letter has been designed to not only interpolate between different scenarios, but also to combine the most generic effects of random dynamics and continuous measurements, and thereby, to further inform the design of suitable experiments. In particular, the unitary dynamics describe the local generation of entanglement by randomly fluctuating interactions, while the employed measurement model describes quantum detection schemes currently employed in solid state nanocircuits [49,51,70-72] and quantum optical devices $[73,74]$. In such settings, the described universality of the entanglement dynamics enhances our understanding of environmental effects, and serves to provide detailed control of the quantum dynamics in simple yet profound ways.

All relevant data present in this publication can be accessed at [75].

This research was funded by the U.K. Engineering and Physical Sciences Research Council (EPSRC) via Grant No. EP/P010180/1. Computer time was provided by Lancaster University's High-End Computing facility. M.S. was also funded by the European Research Council (ERC) under the European Union's Horizon 2020 Research and Innovation programme (Grant agreement No. 853368). 
*mszynisz@gmail.com

[1] A. Degasperis, L. Fonda, and G. C. Ghirardi, Nuovo Cimento A 21, 471 (1974).

[2] B. Misra and E. C. G. Sudarshan, J. Math. Phys. (N.Y.) 18, 756 (1977).

[3] A. Peres, Am. J. Phys. 48, 931 (1980).

[4] Y. Li, X. Chen, and M. P. A. Fisher, Phys. Rev. B 98, 205136 (2018).

[5] A. Chan, R. M. Nandkishore, M. Pretko, and G. Smith, Phys. Rev. B 99, 224307 (2019).

[6] B. Skinner, J. Ruhman, and A. Nahum, Phys. Rev. X 9 , 031009 (2019).

[7] Y. Li, X. Chen, and M. P. A. Fisher, Phys. Rev. B 100, 134306 (2019).

[8] M. Szyniszewski, A. Romito, and H. Schomerus, Phys. Rev. B 100, 064204 (2019).

[9] C.-M. Jian, Y.-Z. You, R. Vasseur, and A. W. W. Ludwig, Phys. Rev. B 101, 104302 (2020).

[10] Y. Li, X. Chen, A. W. W. Ludwig, and M. P. A. Fisher, arXiv:2003.12721.

[11] X. Chen, Y. Li, M. P. A. Fisher, and A. Lucas, Phys. Rev. Research 2, 033017 (2020).

[12] S. Choi, Y. Bao, X.-L. Qi, and E. Altman, Phys. Rev. Lett. 125, 030505 (2020).

[13] M. J. Gullans and D. A. Huse, Phys. Rev. X 10, 041020 (2020).

[14] W.-T. Kuo, A. A. Akhtar, D. P. Arovas, and Y.-Z. You, Phys. Rev. B 101, 224202 (2020).

[15] A. Nahum and B. Skinner, Phys. Rev. Research 2, 023288 (2020).

[16] S. Roy, J. T. Chalker, I. V. Gornyi, and Y. Gefen, Phys. Rev. Research 2, 033347 (2020).

[17] A. Zabalo, M. J. Gullans, J. H. Wilson, S. Gopalakrishnan, D. A. Huse, and J. H. Pixley, Phys. Rev. B 101, 060301(R) (2020).

[18] L. Zhang, J. A. Reyes, S. Kourtis, C. Chamon, E. R. Mucciolo, and A.E. Ruckenstein, Phys. Rev. B 101, 235104 (2020).

[19] R. Fan, S. Vijay, A. Vishwanath, and Y.-Z. You, arXiv: 2002.12385.

[20] Y. Bao, S. Choi, and E. Altman, Phys. Rev. B 101, 104301 (2020).

[21] A. Bera and S. S. Roy, arXiv:2003.12546.

[22] A. Lavasani, Y. Alavirad, and M. Barkeshli, arXiv: 2004.07243.

[23] S. Sang and T. H. Hsieh, arXiv:2004.09509.

[24] M. Ippoliti, M. J. Gullans, S. Gopalakrishnan, D. A. Huse, and V. Khemani, arXiv:2004.09560.

[25] Q. Tang and W. Zhu, Phys. Rev. Research 2, 013022 (2020).

[26] D. Rossini and E. Vicari, Phys. Rev. B 102, 035119 (2020).

[27] S. Goto and I. Danshita, Phys. Rev. A 102, 033316 (2020).

[28] Y. Fuji and Y. Ashida, Phys. Rev. B 102, 054302 (2020).

[29] K. Snizhko, P. Kumar, and A. Romito, Phys. Rev. Research 2, 033512 (2020).

[30] V. Gebhart, K. Snizhko, T. Wellens, A. Buchleitner, A. Romito, and Y. Gefen, Proc. Natl. Acad. Sci. U.S.A. 117, 5706 (2020).

[31] M. J. Gullans and D. A. Huse, Phys. Rev. Lett. 125, 070606 (2020).
[32] J. Lopez-Piqueres, B. Ware, and R. Vasseur, Phys. Rev. B 102, 064202 (2020).

[33] O. Shtanko, Y. A. Kharkov, L. P. García-Pintos, and A. V. Gorshkov, arXiv:2004.06736.

[34] K. Noh, L. Jiang, and B. Fefferman, Quantum 4, 318 (2020).

[35] B.-L. Fang, J. Chen, F. Chen, and L. Ye, Laser Phys. Lett. 17, 085203 (2020).

[36] O. Alberton, M. Buchhold, and S. Diehl, arXiv:2005.09722.

[37] O. Lunt and A. Pal, Phys. Rev. Research 2, 043072 (2020).

[38] X. Turkeshi, R. Fazio, and M. Dalmonte, Phys. Rev. B 102, 014315 (2020).

[39] Y. Li and M. P. A. Fisher, arXiv:2007.03822.

[40] M. Žnidarič, T. Prosen, and P. Prelovšek, Phys. Rev. B 77, 064426 (2008).

[41] J. Eisert, M. Cramer, and M. B. Plenio, Rev. Mod. Phys. 82, 277 (2010).

[42] J. H. Bardarson, F. Pollmann, and J. E. Moore, Phys. Rev. Lett. 109, 017202 (2012).

[43] B. Bauer and C. Nayak, J. Stat. Mech. (2013) P09005.

[44] J. A. Kjäll, J. H. Bardarson, and F. Pollmann, Phys. Rev. Lett. 113, 107204 (2014).

[45] D. J. Luitz, N. Laflorencie, and F. Alet, Phys. Rev. B 91, 081103(R) (2015).

[46] J. R. Petta, A. C. Johnson, J. M. Taylor, E. A. Laird, A. Yacoby, M. D. Lukin, C. M. Marcus, M. P. Hanson, and A. C. Gossard, Science 309, 2180 (2005).

[47] D. Kim, Z. Shi, C. Simmons, D. Ward, J. Prance, T. S. Koh, J. K. Gamble, D. Savage, M. Lagally, M. Friesen et al., Nature (London) 511, 70 (2014).

[48] A. West, B. Hensen, A. Jouan, T. Tanttu, C.-H. Yang, A. Rossi, M. F. Gonzalez-Zalba, F. Hudson, A. Morello, D. J. Reilly et al., Nat. Nanotechnol. 14, 437 (2019).

[49] K. Murch, S. Weber, C. Macklin, and I. Siddiqi, Nature (London) 502, 211 (2013).

[50] P. Neumann, J. Beck, M. Steiner, F. Rempp, H. Fedder, P. R. Hemmer, J. Wrachtrup, and F. Jelezko, Science 329, 542 (2010).

[51] D. Aasen, M. Hell, R. V. Mishmash, A. Higginbotham, J. Danon, M. Leijnse, T. S. Jespersen, J. A. Folk, C. M. Marcus, K. Flensberg, and J. Alicea, Phys. Rev. X 6, 031016 (2016).

[52] J. Manousakis, C. Wille, A. Altland, R. Egger, K. Flensberg, and F. Hassler, Phys. Rev. Lett. 124, 096801 (2020).

[53] J. F. Steiner and F. von Oppen, Phys. Rev. Research 2, 033255 (2020).

[54] M. I. K. Munk, R. Egger, J. Schulenborg, and K. Flensberg, Phys. Rev. Research 2, 033254 (2020).

[55] P. Maioli, T. Meunier, S. Gleyzes, A. Auffeves, G. Nogues, M. Brune, J. M. Raimond, and S. Haroche, Phys. Rev. Lett. 94, 113601 (2005).

[56] X. Cao, A. Tilloy, and A. D. Luca, SciPost Phys. 7, 24 (2019).

[57] J. Napp, R. L. L. Placa, A. M. Dalzell, F. G. S. L. Brandao, and A. W. Harrow, arXiv:2001.00021.

[58] T. Zhou and A. Nahum, Phys. Rev. X 10, 031066 (2020).

[59] A. Nahum, J. Ruhman, S. Vijay, and J. Haah, Phys. Rev. X 7, 031016 (2017). 
[60] C. W. von Keyserlingk, T. Rakovszky, F. Pollmann, and S. L. Sondhi, Phys. Rev. X 8, 021013 (2018).

[61] A. Nahum, S. Vijay, and J. Haah, Phys. Rev. X 8, 021014 (2018).

[62] L. Hua, Harmonic Analysis of Functions of Several Complex Variables in the Classical Domains (American Mathematical Society, Providence, RI, 1963).

[63] The Cauchy process arises from the Lorentzian distribution of $H_{\text {eff }}$; see, e.g., Ref. [64]. An analogous entanglement dynamics can be obtained from a Wiener process where $H_{\text {eff }}$ is taken from the Gaussian unitary ensemble with matrix elements $H_{\text {eff, } l m}=\mathrm{O}(1 / \mu)$; for more details see Ref. [65].

[64] P. W. Brouwer, Phys. Rev. B 51, 16878 (1995).

[65] See Supplemental Material at http://link.aps.org/ supplemental/10.1103/PhysRevLett.125.210602 for an analysis of the transition in terms of the bipartite information, a discussion of the role of measurement frequency, the description and further discussion of the finite-size scaling, and a comparison between different stochastic processes that can be used to approach the continuum limit. This Supplemental Material also includes a reference to [66].
[66] F. Haake, M. Kus, H.-J. Sommers, H. Schomerus, and K. Zyczkowski, J. Phys. A 29, 3641 (1996).

[67] P. A. Mello, P. Pereyra, and T. H. Seligman, Ann. Phys. (N.Y.) 161, 254 (1985).

[68] K. Jacobs, Quantum Measurement Theory and Its Applications (Cambridge University Press, Cambridge, England, 2014).

[69] H. M. Wiseman and G. J. Milburn, Quantum Measurement and Control (Cambridge University Press, Cambridge, England, 2009).

[70] M. Field, C. G. Smith, M. Pepper, D. A. Ritchie, J. E. F. Frost, G. A. C. Jones, and D. G. Hasko, Phys. Rev. Lett. 70, 1311 (1993).

[71] A. N. Korotkov, Phys. Rev. B 60, 5737 (1999).

[72] A. Romito, Y. Gefen, and Y. M. Blanter, Phys. Rev. Lett. 100, 056801 (2008).

[73] O. Hosten and P. Kwiat, Science 319, 787 (2008).

[74] P. B. Dixon, D. J. Starling, A. N. Jordan, and J. C. Howell, Phys. Rev. Lett. 102, 173601 (2009).

[75] https://doi.org/10.17635/lancaster/researchdata/396. 